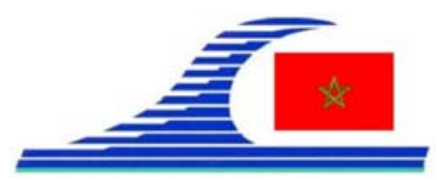

Conférence Méditerranéenne Côtière et Maritime

EDITION 2, TANGER, MAROC (2011)

Coastal and Maritime Mediterranean Conference

Disponible en ligne - $h$ ttp://www.paralia.fr - Available online

\title{
Décontamination des sédiments marins pollués en métaux lourds par extraction en milieu complexant
}

\author{
Fatiha OUDGHIRI ${ }^{1}$, M $^{\text {a }}$ Rocío RODRÍGUEZ BARROSO ${ }^{1}$, \\ José Luis GARCÍA MORALES ${ }^{1}$, Bouchta EL MOUMNI ${ }^{2}$
}

1 Departamento Tecnologías del Medio Ambiente, Universidad de Cádiz, Facultad de Ciencias del Mar y Ambientales, Edificio CASEM, Polígono Río San Pedro, España. fatiha.oudghiri@uca.es

2 Département des Sciences de la Terre et d'Océanologie, Université Abdelmalek Essaâdi, Faculté des Sciences et Techniques, BP 416, Tanger, Maroc.

\section{Résumé :}

La présente étude de recherche vise à décontaminer des sédiments portuaires de la baie de Cadix contaminés en métaux lourds par un procédé d'extraction chimique.

Nous avons étudié, en utilisant des modes opératoires adaptés, l'extraction du $\mathrm{Cu}, \mathrm{Zn}$ et $\mathrm{du} \mathrm{Pb}$ en présence de complexants. L'étude a été réalisée sur deux types de sédiments contaminés par une activité industrielle et nautique. Nous avons comparé dans un milieu acide l'efficacité du réactif complexant EDTA en $0,1 \mathrm{M}$ et en $0,3 \mathrm{M}$. Les résultats montrent que l'EDTA 0,3M est le plus performant pour extraire respectivement le $\mathrm{Pb}$, $\mathrm{Cu}$ et le $\mathrm{Zn}$.

Le rendement d'extraction observé pour l'EDTA 0,3M était de $69 \%$ pour le plomb, $58 \%$ pour le cuivre, et de $48 \%$ pour le zinc

\section{Mots-clés :}

Sédiment - Extraction - Métaux lourds - Complexant

\section{Introduction}

L'extraction chimique par ajout d'un agent complexant constitue une approche pour l'extraction des métaux lourds des sédiments contaminés en formant des complexes métalliques solubles. En effet, les chélateurs sont des composés ayant la propriété de se lier fortement et de manière plus ou moins spécifique à un ion positif bivalent et trivalent (notamment métallique) avec lequel ils forment un composé (ou chélate) dans lequel l'atome central est lié aux atomes voisins par au moins deux liaisons en formant une structure annulaire.

L'acide éthylènediaminététracétique (EDTA), l'acide nitrilotriacétique (NTA) et l'acide Diéthylènetriaminepentaacétique (DTPA) sont les principaux agents chélateurs ayant été testés pour l'enlèvement des métaux lourds (LEGIEC, 1997 ; FISHER et al., 1998 ; RAMPLEY \& OGDEN,1998; SAMANI et al.,1998).

$\mathrm{Au}$ cours de notre étude, nous avons utilisé le complexant hexadentate EDTA, largement utilisé pour des applications industrielles, comme agent complexant dans les 
La connaissance de la Mer :

un vecteur du développement durable en Méditerranée

lessives. Il est aussi utilisé pour éviter la formation de précipités, pour extraire un métal d'un système et pour garder un métal disponible en solution. (BUCHELI-WITSCHEL \& EGLI, 2001).

Ce complexant a été testé avec succès par MCCREADY et al. (2003). pour extraire le $\mathrm{Pb}, \mathrm{Cu}$ et le $\mathrm{Zn}$ présents dans les sédiments portuaires. Ce complexant a également été testé par ELLIOT et BROWN (1989) pour éliminer le plomb présent dans les sols contaminés.

\section{Matériels et méthodes}

\section{1 Échantillonnage du sédiment}

Les sédiments étudiés $\left(\mathrm{S}_{1}\right.$ et $\left.\mathrm{S}_{2}\right)$ ont été prélevés dans la baie de Cadix près d'une zone où l'on fabrique des navires à l'aide d'une benne de type Van Veen, en acier inoxydable (figure 1).

$\mathrm{Au}$ laboratoire, les échantillons de sédiments ont été mis à l'étuve $\left(50^{\circ} \mathrm{C}\right)$ jusqu'à séchage complet. Ils ont été ensuite broyés à $63 \mu \mathrm{m}$ et homogénéisés dans un mortier en agate manuel. La concentration en métaux a été déterminée par ICP-AES (Inductively Coupled Atomic Emission Spectrometry) ou par ICP-MS (Inductively coupled plasma mass spectroscopy). Le protocole de dosage des métaux lourds a été validé sur des matériaux de référence certifiés (MESS-3 et PACS-2).

\subsection{Protocole d'extraction des métaux lourds}

L'ensemble des expériences ont été réalisées sur deux types de sédiments contaminés, séchés à l'air, désagrégés et tamisés à $2 \mathrm{~mm}$. Le rapport solide/ liquide utilisé est de 1/10. L'extraction a été effectuée en mélangeant $50 \mathrm{ml}$ de la solution complexante avec $5 \mathrm{~g}$ de sédiment, sous agitation magnétique continu pendant $2 \mathrm{~h}$ à température ambiante. Le mélange a été ensuite centrifugé à $4000 \mathrm{tr} / \mathrm{mn}$ pendant $15 \mathrm{~min}$ et filtré sur un filtre de porosité $0,45 \mu \mathrm{m}$ et dosé par ICP-AES ou par ICP-MS.

\section{Résultats}

Les pourcentages de plomb, de cuivre et de zinc extraits du sédiment $S_{1}$ et $S_{2}$ au milieu acide sont présentés sur les figures suivantes :

Après un temps de contact de $2 \mathrm{~h}$ à $\mathrm{pH}=3,8$ et un rapport solide/liquide de $1 / 10$. Les résultats des extractions (figure 2,3) montrent que l'EDTA 0,3 $\mathrm{M}$ est plus efficace que l'EDTA 0.1 M pour extraire respectivement le $\mathrm{Pb}, \mathrm{Cu}$ et le $\mathrm{Zn}$.

Les résultats obtenus pour les deux types de sédiments ont montré que le plomb est plus facile à extraire que le cuivre et que ce dernier est plus facile à extraire que le zinc. Ces résultats, sont similaires à ceux trouvés par Leleyter et Baraud(2005).

Le maximum d'extraction des métaux pour l'EDTA 0,3M était de $69 \%$ pour le plomb, $58 \%$ pour le cuivre et $48 \%$ pour le zinc. 
A best knowledge of the Sea: A sustainable development vector in Mediterranean

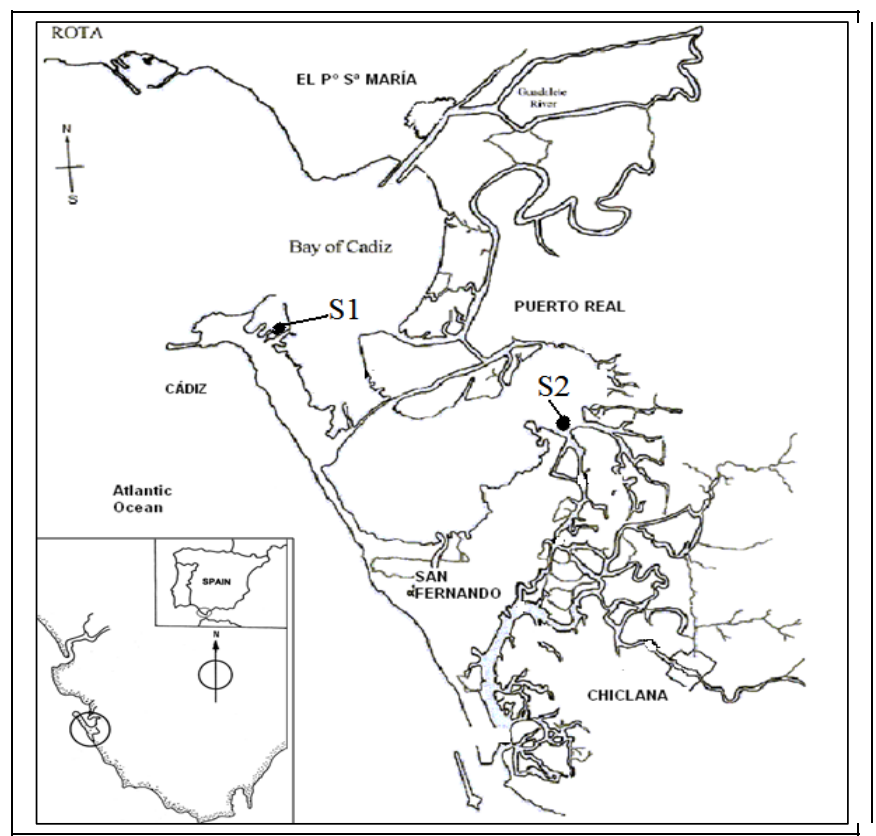

Fig. 1. Situation géographique et localisation de station de prélèvements dans la baie de Cadix

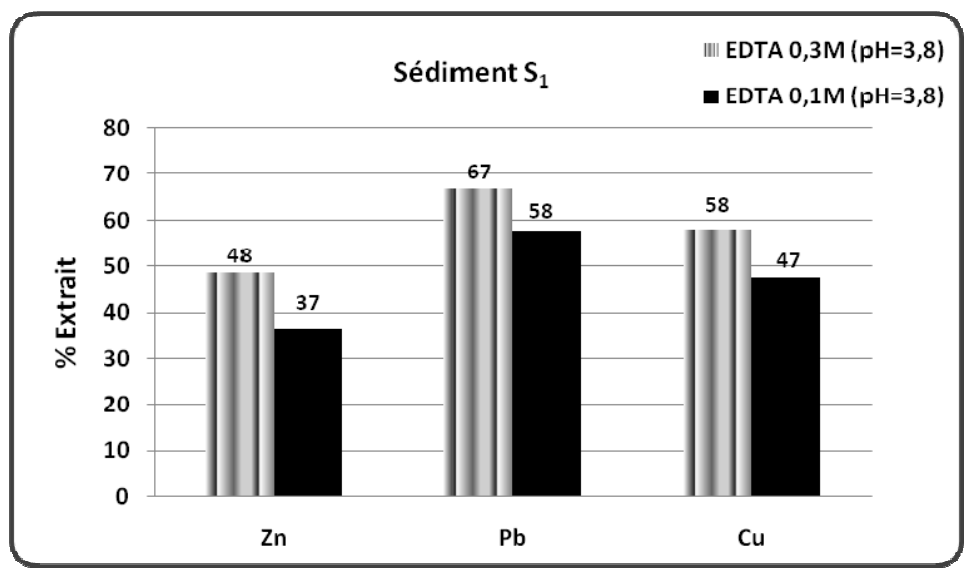

Fig. 2. Extraction des métaux lourds par l'EDTA et en milieu acide dans le sédiment $S_{1}$

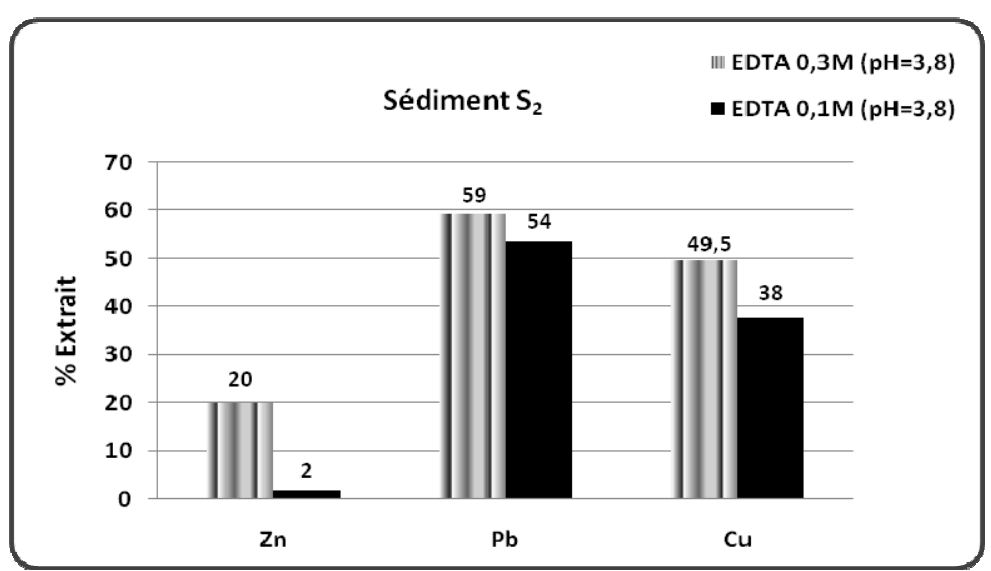

Fig. 3. Extraction des métaux lourds par l'EDTA et en milieu acide dans le sédiment $S_{2}$. 
La connaissance de la Mer :

un vecteur du développement durable en Méditerranée

Les rendements d'extraction sont moins élevés pour le sédiment S2. La différence de rendement d'extraction observable entre les deux échantillons de sédiment est probablement liée à des sources différentes de contamination, propres à chacun des deux sites.

\section{Conclusions}

Les résultats obtenus lors de cette étude, ont montré que l'EDTA $0,3 \mathrm{M}$ possède un pouvoir complexant très important permettant d'extraire une grande partie du $\mathrm{Pb}, \mathrm{Cu}$ et du $\mathrm{Zn}$ dans les sédiments marins contaminés puisque le rendement d'extraction observé était de $69 \%$ pour le plomb, $58 \%$ pour le cuivre, et de $48 \%$ pour le zinc.

Remerciements : Les auteurs remercient l'Agence Espagnole de Coopération Internationale (AECI) qui a financé ce projet de recherche dans le cadre du programme interuniversitaire de coopération entre l'Espagne et le Maroc, projet "Técnicas termogravimétricas como herramienta de control de contaminación de sedimentos: (1) aplicación a Planes de Vigilancia Ambiental, (2) técnica de control en descontaminación de sedimentos" $\mathrm{n}^{\mathrm{o}} \mathrm{A} / 023497 / 09$ et le projet "Unidad para la gestión sostenible de residuos en el Norte de Marruecos" nº A1/039099/11.

\section{Références bibliographiques}

BUCHELI-WITSCHEL M., EGLI T. (2001). Environmental fate and microbial degradation of aminopolycarboxylic acids. FEMS Microbiology Reviews 25, pp 69-106. doi:10.1111/j.1574-6976.2001.tb00572.x

ELLIOT H.A., BROWN G.A. (1989). Comparative evaluation of NTA and EDTA for extractive decontamination of Pb-polluted soils. Water Air Soil Pollut. 45, pp 361-369. doi:10.1007/BF00283464

FISHER K., BIPP H.P., RIEMSCHNEIDER P., LEIDMANN P., BIENIEK D., KETTRUP A. (1998). Utilization of biomass residues for the remediation of metalpolluted soils. Environ. Sci. Technol., 32, pp 2154-2161. doi:10.1021/es9706209

LEGIEC I.A. (1997). Pb mobility and extractant optimization for contaminated soils. Environ. Prog., 16, pp 88-92. doi:10.1002/ep.3300160213

RAMPLEY C.G., OGDEN K.L. (1998). Preliminary studies for removal of lead from surrogate and real soils using a water soluble chelator: adsorption and batch extraction. Environ. Sci. Technol., 32, pp 987-993. doi:10.1021/es9706256

MCCREADY S., BIRCH G.F., TAYLOR S.E. (2003). Extraction of heavy metals in Sydney Harbour sediments using $1 \mathrm{M} \mathrm{HCl}$ and 0.05M EDTA and implications for sediment-quality guidelines. Australian Journal of Earth Sciences 50, pp 249-255.

SAMANI Z., HU S., HANSON A.T., HEIL D.M. (1998). Remediation of lead contaminated soil by column extraction with EDTA: II. modeling. Water Air Soil Pollut.,102, pp 221-238. doi:10.1023/A:1004901013628 